\title{
Touching ghosts: observing free fall from an infalling frame of reference into a Schwarzschild black hole
}

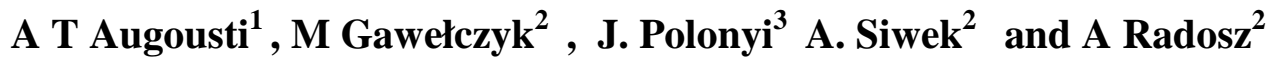 \\ ${ }^{1}$ Faculty of Science, Kingston University, Kingston, Surrey KT1 2EE UK \\ ${ }^{2}$ Institute of Physics, Wroclaw University of Technology, Wybrzeze Wyspianskiego 27, 50370 \\ Wroclaw, Poland \\ ${ }^{3}$ University of Strasbourg, High Energy Physics Theory Group, CNRS-IPHC, 23 rue de Loess, \\ BP28 67037 Strasbourg Cedex 2, France
}

Email: augousti@kingston.ac.uk

\begin{abstract}
.
The problem of communication between observers in the vicinity of a black hole in a Schwarzschild metric is considered. The classic example of an infalling observer Alice and a static distant mothership MS is extended to include a second infalling observer Bob, who follows Alice in falling towards the event horizon. Kruskal coordinates are introduced to analyze this situation, and the pedagogic value of introducing this alternative coordinate system is demonstrated by their ability to provide a graphically based solution and illustration to this problem. The counterintuitive result that is obtained, which will be of interest to those studying general relativity at an introductory level at final year undergraduate or at graduate level, is that Bob appears to reach a ghostly image of Alice at the event horizon.
\end{abstract}

\section{Introduction}

One of the most appealing features of the gravitational field yielded by a spherically symmetric, static mass $\mathrm{M}$, is the fact that it is an exactly solvable problem both in classical and in relativistic mechanics. The meaning of exact solvability is that the gravitational field itself is known (in relativistic mechanics, the metric tensor) and the equation of motion of a test particle can be solved. That is why it is often used as an example of a relativistic equation of motion in the framework of Schwarzschild coordinates. The simplest problem in this case is radial free fall. Despite the exact solvability and simplicity of the problem (see e.g. [1]) one can still find some new, unexpected, and even intriguing features. One such unexpected characteristic (unknown to the best of our knowledge) occurs when discussing the problem of communication with an object falling towards the event horizon in this case. A well-known feature of radial fall towards the event horizon is that the coordinate time (the time that would be measured by a static distant observer) taken to reach the event horizon by a freely falling object is infinite (see e.g. [2]). This feature is accompanied by unlimited redshift: the frequency ratio of electromagnetic signals coming from the falling object and recorded by a distant observer at rest, tends to zero as the infalling observer approaches the event horizon. In principle, such signals will be recorded for an infinite future duration; in practice, they will disappear from the screens of rest observers due to the sensitivity limits of recording devices. On the other hand, an infalling observer reaches the event horizon in a finite proper time (the time measured by the infalling observer). This may be regarded as the most dramatic manifestation of time-dilation-like phenomena [2].

Bearing in mind such a dramatic difference between the measurements of an observer at rest and an infalling observer, one may consider an intermediate situation, and ask: what would be 
the observations made by two infalling observers, A (Alice) being followed by B (Bob)? Alice crosses the event horizon in "the infinite future" in terms of coordinate time, but in a finite proper time, as measured by her - what would be Bob's perception of this event? How much later (behind Alice) will Bob himself cross the event horizon, according to his proper time? Would this be shortly after Alice appears to? We will discuss these questions here because the key answer turns out, to be rather surprising: Bob, while continuously in receipt of signals from Alice, finds her crossing the horizon, at the instant that he himself crosses the horizon. This counter-intuitive result is found to be much less surprising when studied in more detail. An especially illuminating description of this effect may be given using so-called Kruskal coordinates $[3,4]$, and it has pedagogic value to introduce the use of these specialized co-ordinates to a wider audience by addressing this particular problem of interest. By focusing primarily on the qualitative character of this observation rather than on its quantitative aspects, one can obtain an appealing and simple description of this nontrivial observation. A further pedagogical value of this approach is added by the fact that although it is not a new, scientifically original result, (referring to objects that in principle have been understood for more than 90 years - Schwarzschild black holes [5,6] - although in practice widely accepted for only some 40 years). Apart from static black holes, there are also charged [6] and/or rotating black holes $[7,8]$, described also in introductory texts $[9,10]$ as well as more rigorous textbooks $[11,12]$. This current description provides a new perspective on a well-known feature of these objects, namely free fall in Schwarzschild spacetime. As a matter of fact, this new perspective might be added to the list of phenomena unknown in the "classical world" that are accessible to us only by means of relativistic physics.

\section{Radial fall in Schwarzschild spacetime}

Let us start by defining the framework for our considerations. One may consider a static, spherically symmetric mass $\mathrm{M}$ of radius $\mathrm{R}$. The properties of the gravitational field outside of this gravitating body, $r>R$, are described in terms of the metric tensor. The metric tensor expressed in Schwarzschild coordinates turns out to be diagonal and the line element is then expressed as:

$$
\begin{aligned}
& d \tau^{2}=\left(1-\frac{r_{S}}{r}\right) c^{2} d t^{2}-\left(1-\frac{r_{S}}{r}\right)^{-1} d r^{2}-r^{2} d \theta^{2}-r^{2} \sin ^{2} \theta d \varphi^{2} \\
& \equiv g_{00}\left(d x^{0}\right)^{2}+g_{11}\left(d x^{1}\right)^{2}+g_{22}\left(d x^{2}\right)^{2}+g_{33}\left(d x^{3}\right)^{2}
\end{aligned}
$$

where the Schwarzschild radius is denoted $r_{S}=\frac{2 G M}{c^{2}}$. Solution (1) holds above the event

horizon, $r>r_{s}$.

The motion of a test particle within a Schwarzschild spacetime (1) may be described in a rigorous manner by reference to conservation laws. There are two conserved quantities, energy and angular momentum. The planar motion (angular momentum conservation), may be considered to occur in an equatorial plane, $\theta=\frac{\pi}{2}$ and hence the equation of motion simplifies in this case to the four-velocity condition

$g_{00}\left(u^{0}\right)^{2}+g_{11}\left(u^{1}\right)^{2}+g_{33}\left(u^{3}\right)^{2}=1$

where, $u^{\alpha}=\frac{d x^{\alpha}}{d \tau}$ denotes the $\alpha$ - component of the velocity vector. 
Hereafter radial fall only is considered, implied by $u^{3}=0$. Then energy conservation, (a result of the time-independence of the system, see [1, 13]) gives an explicit dependence for the time component velocity vector

$$
u_{0}=g_{00} u^{0}=\varepsilon
$$

This in turn (see Eq. 2) leads to the obvious solution for the radial velocity component: $u^{1}=\mp \sqrt{\varepsilon^{2}-g_{00}}$

The parameter $\varepsilon=\sqrt{g_{00}\left(r_{0}\right)}$ can be shown to be related to the energy, and is determined by the initial conditions: $r_{0}$ denotes the starting point of the radial fall, assuming free fall beginning from rest. One can also consider massless test particles such as photons. In this case the corresponding tangential four vector $k^{\alpha}=\frac{d x^{\alpha}}{d \sigma}$ is, (by definition) a null vector,

$g_{\alpha \beta} k^{\alpha} k^{\beta}=0$

where $\sigma$ is an auxiliary parameter of a photon world line. Components of the wave vector for radial fall, found by use of the energy conservation condition (c.f. Eq. 3a) and Eq. 4, take the form (see the discussion in Ref. [14]):

$k^{0}=\frac{\omega_{\infty}}{g_{00}} \quad k^{1}=\mp \omega_{\infty}$

The $\mp$ sign corresponds to incoming and outgoing massless test particles (light rays).

By using Eqs. (3) one can calculate the time elapsed to reach the event horizon. The proper time, i. e. the time as measured by the infalling observer is defined by Eq. (3b),

$$
d \tau=-\frac{d r}{\sqrt{g_{00}\left(r_{0}\right)-g_{00}(r)}}=-\frac{d r}{\sqrt{\frac{r_{S}}{r}-\frac{r_{S}}{r_{0}}}}
$$

while the coordinate time is defined by eqs. (3a) and (3b)

$$
d t=-\frac{\sqrt{g_{00}\left(r_{0}\right)}}{\left(1-\frac{r_{S}}{r}\right) \sqrt{g_{00}\left(r_{0}\right)-g_{00}(r)}} d r=-\frac{\sqrt{1-\frac{r_{S}}{r_{0}}}}{\left(1-\frac{r_{S}}{r}\right) \sqrt{\frac{r_{S}}{r}-\frac{r_{S}}{r_{0}}}} d r
$$

As direct calculation shows $[2,15]$ an in-falling object_reaches the event horizon

a) after a finite amount of proper time (according to the infalling observer), and

b) after an infinite time (according to a distant, static observer)

Indeed, by using the substitution $x=\sqrt{\frac{r_{0}}{r}-1}$ one can integrate Eq. (6a)

$$
\tau-\tau_{0}=-\int_{r_{0}}^{r} \frac{d \bar{r}}{\sqrt{\frac{r_{S}}{\bar{r}}-\frac{r_{S}}{r_{0}}}}=r_{0} \sqrt{\frac{r_{0}}{r_{S}}}\left(\arctan x+\frac{x}{1+x^{2}}\right),
$$

to find that in-falling observer starting from $r_{0}$ reaches the event horizon in a finite (proper) time, 


$$
\begin{aligned}
& \Delta \tau=\sqrt{\frac{r_{0}}{r_{S}}}\left(r_{0} \arctan x_{S}+r_{S} x_{S}\right)<\sqrt{\frac{r_{0}}{r_{S}}}\left(r_{0} \frac{\pi}{2}+r_{S} x_{S}\right), \\
& x_{S}=\sqrt{\frac{r_{0}}{r_{S}}-1} .
\end{aligned}
$$

On the other hand, the coordinate time period, found from Eq. (6 b),

$$
t-t_{0}=-\int_{r_{0}}^{r} \frac{d \bar{r}}{\left(1-\frac{r_{S}}{\bar{r}}\right) \sqrt{\frac{r_{S}}{\bar{r}}-\frac{r_{S}}{r_{0}}}}=-r_{S}\left[\ln \left(x_{S}-x\right)-\ln \left(x_{S}+x\right)\right]+2 r_{S} x_{S} \arctan x+r_{S} x_{S}\left(\arctan x+\frac{x}{1+x^{2}}\right)
$$

tends to infinity as $r$ approaches the event horizon,

$\Delta t \propto-r_{S} \ln \left(x_{S}-x\right) \stackrel{x \rightarrow x_{S}}{\longrightarrow} \infty$.

This is a time-dilation-like effect and it is a well-known feature of a Schwarzschild black hole. Less well-known is the frequency ratio relation accompanying mutual signal exchange between Alice and her "mother station", MS located at $r_{0}$. Namely one finds that the frequency ratio is redshifted in both cases. MS receives (r) signals emitted (e) by Alice, and the frequency ratio defined as a scalar product ratio [16].

$$
\frac{\omega_{M S}^{r}}{\omega_{A}^{e}}=\frac{\left(k^{\alpha} u_{\alpha}\right)_{M S}}{\left(k^{\alpha} u_{\alpha}\right)_{A}}=\frac{\left(g_{00} k^{0} u^{0}\right)_{M S}}{\left(g_{00} k^{0} u^{0}+g_{11} k^{1} u^{1}\right)_{A}}=\frac{\frac{\omega_{\infty}}{\sqrt{g_{00}\left(r_{0}\right)}}}{\omega_{\infty} u^{0}\left(1+\frac{g_{11} u^{1}}{u^{0}}\right)_{A}}
$$

This may be expressed in terms of Alice's local velocity, $v_{A}$ (as measured by a local observer at rest [17]), via

$$
u_{A}^{0}=\frac{1}{\sqrt{g_{00}\left(r_{A}\right)}} \frac{1}{\sqrt{\left(1-\frac{v_{A}^{2}}{c^{2}}\right)}}
$$

Then it takes a simple form, namely

$$
\frac{\omega_{M S}^{r}}{\omega_{A}^{e}}=1-\frac{v_{A}}{c}
$$

and is critically redshifted, i.e. it tends to zero [18], because $v_{A}$ tends to $\mathrm{c}$ as the event horizon is approached. (There is a simple argument that $v_{A}$ tends to $\mathrm{c}$ independently of the starting point $r_{0}$ : an immediate consequence of the energy conservation (3a) and Eq. (9a) is that

$$
\frac{\sqrt{g_{00}\left(r_{A}\right)}}{\sqrt{\left(1-\frac{v_{A}^{2}}{c^{2}}\right)}}=\mathrm{const}
$$

is conserved, hence $v_{A}$ tends to $\mathrm{c}$ when $r_{A} \rightarrow r_{S}$ [17]). In turn Alice receives signals issued by MS, universally redshifted, 


$$
\frac{\omega_{A}^{r}}{\omega_{M S}^{e}}=\frac{1}{1+\frac{v_{A}}{c}}
$$

This ratio tends to $1 / 2$ when Alice approaches the event horizon, independently of the starting position. This asymmetry in communication eventually becomes singular: MS receives Alice's signals emitted only above the event horizon, whereas Alice will receive signals from MS continuously above and below the event horizon. In this sense two-way communication is disrupted. One may ask how would two-way communication be disrupted between two falling observers, Alice and Bob, with Bob following Alice. Both Alice and Bob would reach the horizon within a finite proper time period so their two-way communication must be broken within a finite proper time.

To study this more easily one needs a change of reference frame. Namely, the considerations above have been performed within Schwarzschild coordinates, which produce a singularity at the event horizon, $r=r_{S}$. A different frame of reference may be deployed, devoid of this defect.

\section{Kruskal coordinates}

Schwarzschild coordinates (1) yield a singularity at the event horizon: $g_{00}\left(r_{S}\right)=0$. This singularity is termed a coordinate singularity and it does not appear in other, carefully chosen systems of coordinates. There are various coordinate systems free of this singularity $[1,2]$. One of them is the Kruskal (or Kruskal-Szekeres) coordinate frame [2 - 4]. Coordinates in this frame are usually denoted as $v, u, \theta, \varphi$ and the relation $(t, r) \rightarrow(v, u)$ is the following:

$$
\begin{aligned}
& v=\left|\frac{r}{2 M}-1\right|^{1 / 2} e^{\frac{r}{4 M}} \begin{cases}\sinh \left(\frac{t}{4 M}\right) & r>r_{S} \\
\cosh \left(\frac{t}{4 M}\right) & r>r_{S}\end{cases} \\
& u=\left|\frac{r}{2 M}-1\right|^{1 / 2} e^{\frac{r}{4 M}} \begin{cases}\cosh \left(\frac{t}{4 M}\right) & r>r_{S} \\
\sinh \left(\frac{t}{4 M}\right) & r>r_{S}\end{cases}
\end{aligned}
$$

The last two coordinates are those of Schwarzschild angular coordinates.

The transformation (11), above the event horizon $r>r_{S}$, is described by the following matrix,

$$
\frac{\partial(v, u)}{\partial(t, r)}=\left[\begin{array}{cc}
\frac{1}{4 M} u & \frac{1}{4 M g_{t t}} v \\
\frac{1}{4 M} v & \frac{1}{4 M g_{t t}} u
\end{array}\right] .
$$

Inverting this matrix results in the inverse transformation: 


$$
\frac{\partial(t, r)}{\partial(v, u)}=D^{-1}\left[\begin{array}{cc}
\frac{1}{4 M g_{t t}} u & -\frac{1}{4 M g_{t t}} v \\
-\frac{1}{4 M} v & \frac{1}{4 M} u
\end{array}\right],
$$

where,

$$
D=\operatorname{det}\left|\frac{\partial(v, u)}{\partial(t, r)}\right|=\frac{1}{16 M^{2} g_{t t}}\left(u^{2}-v^{2}\right)=\frac{r}{32 M^{3}} e^{\frac{r}{2 M}}
$$

By performing transformation $(12 b)$ one can find the corresponding metric tensor in Kruskal coordinates

$$
K_{\gamma \delta}=g_{\alpha \beta} \frac{\partial x^{\alpha}}{\partial y^{\gamma}} \frac{\partial x^{\beta}}{\partial y^{\delta}},
$$

that does not reveal singular behavior. In fact the line element, expressed in Kruskal coordinates, is

$d \tau^{2}=K\left(d v^{2}-d u^{2}\right)-r^{2} d \theta^{2}-r^{2} \sin ^{2} \theta d \varphi^{2}$

where $K=\frac{32 M^{3}}{r} e^{-\frac{r}{2 M}}$.

The most appealing features of Kruskal coordinates are: the nonsingular behaviour of the metric tensor and the fact that radial geodesics, in- and outgoing, are represented in the v-u plane as perpendicular, straight lines at $-45^{\circ}$ and $+45^{\circ}$, respectively (see below). The meaning of $\mathrm{v}$ - $\mathrm{u}$ coordinates becomes clearer when considering specific hypersurfaces - lines in this case (see Fig.1). 


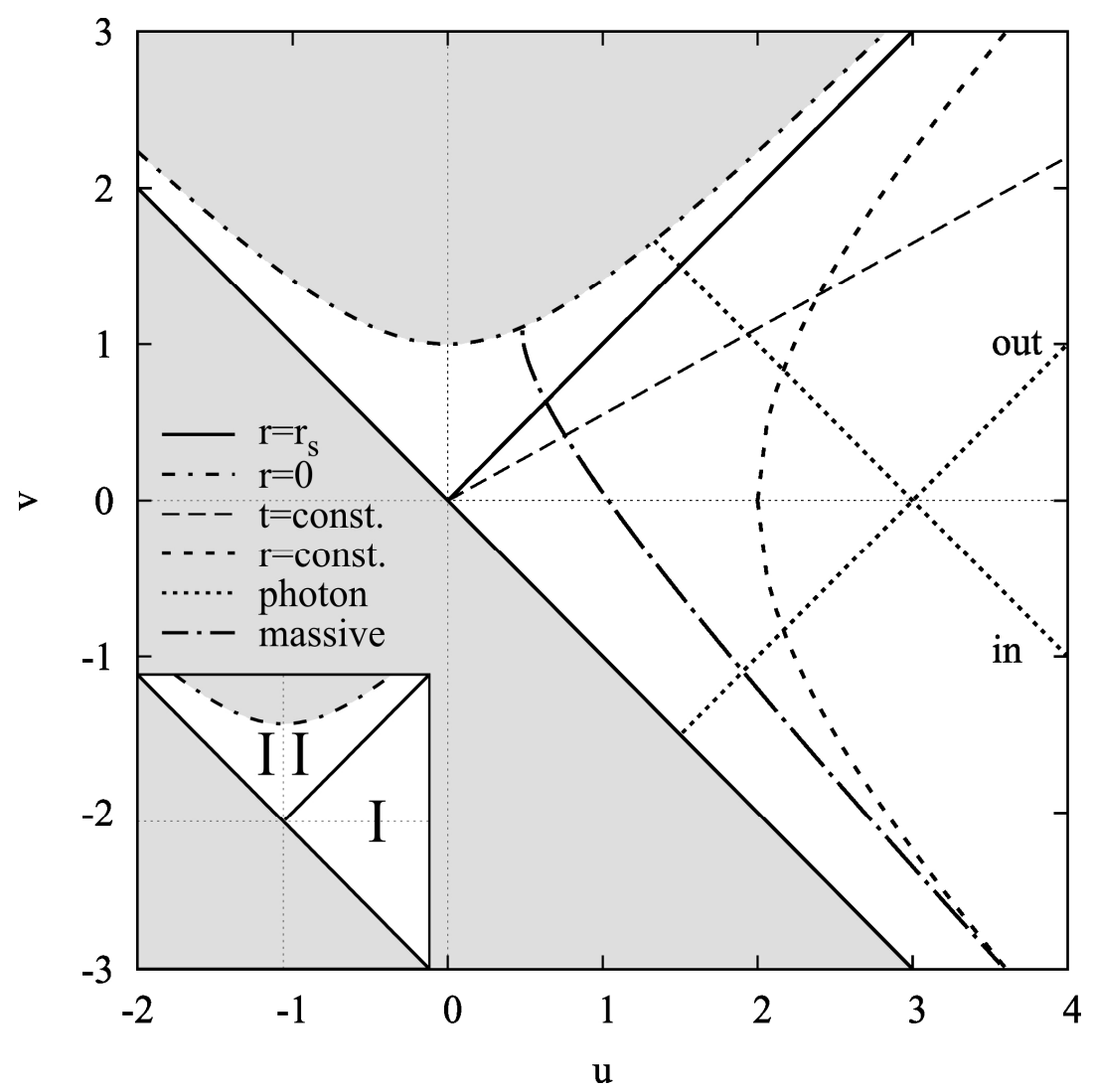

Fig.1 Kruskal coordinates v-u and radial geodesics. Manifold $u+v>0$, covers black hole exterior (I) and interior (II). Hyperbolas with asymptotes $u= \pm v$ represent "surfaces" of constant radius $r$; straight lines, passing through the origin, represent "surfaces" of constant time $t$. Ingoing and outgoing radial null geodesics are $\mp 45^{\circ}$ straight lines. A geodesic for a massive particle is sketched schematically

Namely, world lines of fixed distance, $r=$ const are hyperbolas, $u^{2}-v^{2}=C$; spacelike curves, $t=$ const are straight lines, originating at $v=u=0$. Therefore, the region (I) bounded in Fig. 1 by the two straight lines: $v=-u$ corresponding to, $r=r_{S}, t=-\infty$ and $v=u$ corresponding to $r=r_{S}, t=\infty$ represents the Schwarzschild coordinate system (1).

In the range $r>r_{S}$, applying transformation (12b) to the solutions (3) and (5) one obtains the equations for geodesics. Geodesics are arranged in the v-u plane and velocity vector components are then given as follows:

$$
\begin{aligned}
U^{v} & =\frac{r}{4 M(r-2 M)}\left(u \varepsilon-v \sqrt{\varepsilon^{2}-\frac{(r-2 M)}{r}}\right) \\
U^{u} & =\frac{r}{4 M(r-2 M)}\left(v \varepsilon-u \sqrt{\varepsilon^{2}-\frac{(r-2 M)}{r}}\right)
\end{aligned}
$$

where $\varepsilon$ is a constant of motion (see Eq. 3). Let us emphasize that the velocity components (15) are well-defined in the whole range $0<r<\infty$. The wave-vector components are:

$$
k_{v}=\frac{4 M \Omega}{u^{2}-v^{2}}(u \mp v)
$$


$k_{u}=-\frac{4 M \Omega}{u^{2}-v^{2}}(v \mp u)$

These null geodesics are straight lines, at $\mp 45^{\circ}$ to x-axis, where the label $\mp$ stands for ingoing (outgoing) rays (see Fig.1).

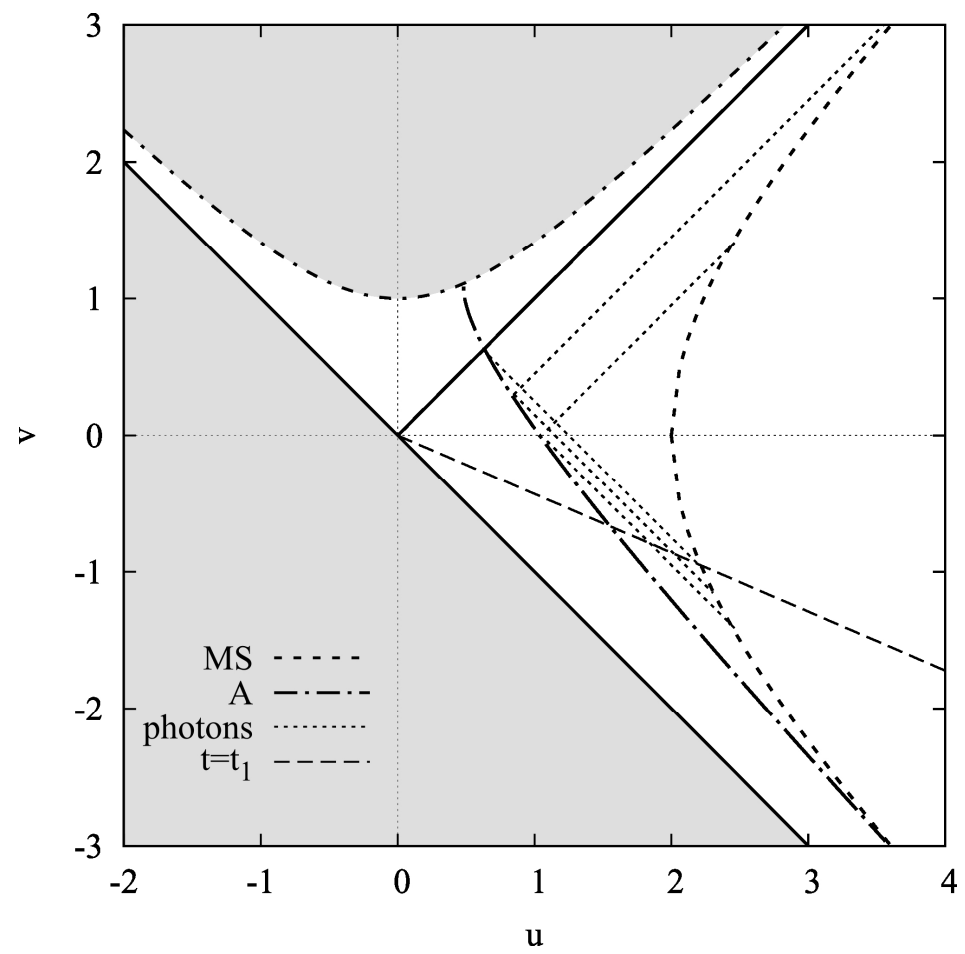

Fig.2 Exchange of radial signals between Alice and "mother station", MS. Alice records (in principle) the signals from MS above and below the horizon; MS records only those signals sent above horizon

Applying these tools to the information exchange between Alice and the mother station MS (see Fig. 2) one can reproduce qualitatively the well-known result: Alice receives messages from MS throughout her journey towards the event horizon and also below the event horizon. MS will receive messages sent by Alice from above the event horizon, throughout all the infinite future. Actually, due to formal technical limitations, these messages, when sufficiently redshifted, will cease to arrive as their intensity will not be sufficiently high.

What may be easily deduced from Fig. 2 is that MS can, in principle, infer the last instant at which a message sent to Alice could produce a response that can be received asymptotically by MS in the future, namely the instant $t=t_{1}$. However, signals from Alice suffer unlimited redshift and as claimed above, at some stage they cease to be recorded by MS.

\section{How does Bob "see" Alice crossing the event horizon?}

One may ask about communication between Alice and Bob, both falling towards the event horizon. As static observers, MS (and all other observers arranged radially at rest), receive messages from Alice continuously throughout the infinite future; the question is, how will observer Bob, who is also going to cross the event horizon in a finite proper time $\tau_{B}$, "see" Alice approaching and crossing the event horizon? Instead of giving a direct answer, let us first consider a trivial question: how does Bob see Alice reaching and crossing a specific 
point at coordinate $r=r_{1}$ ? If Alice and Bob are communicating by means of electromagnetic signals then, Alice, when crossing $r=r_{1}$, at some $t=t_{1}$ sends a signal (back) to Bob. In Kruskal coordinates this signal is a straight line, $+45^{\circ}$ that hits Bob's world line at some later instant $t=t_{2}$, at $r=r_{2}$ (see Fig.3). One can use proper time notation: Alice's and Bob's world lines may be parameterized with their own proper times $\tau^{A}$ and $\tau^{B}$ respectively, and consequently respective proper times representing the crossing instant $t=t_{1}$ (emission instant) and receiving instant $t=t_{2}$ would be fixed as $\tau_{1}^{A}$ and $\tau_{2}^{B}$, respectively.

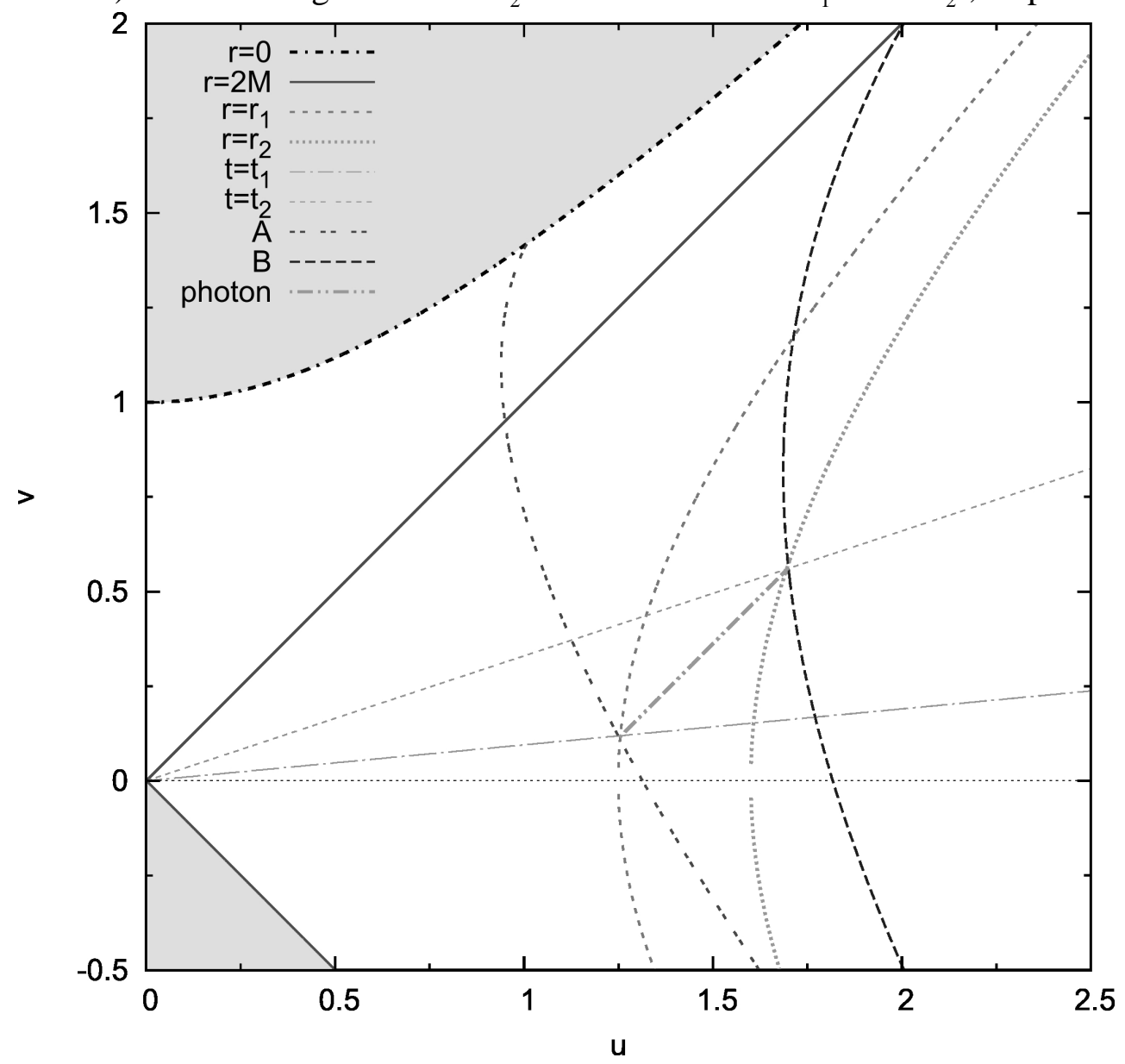

Fig.3 Bob receives signal at $t=t_{2}, r=r_{2}$ from Alice crossing $r=r_{1}$ at $t=t_{1}$.

The situation of crossing the event horizon is, however, a special case. Approaching the event horizon Alice emits signals continuously: those signals reach Bob continuously. But as can be seen in the Fig. 4 those signals are recorded by Bob, who is also approaching the event horizon, until the very instant of crossing the event horizon himself! It looks like information that Alice crosses the event horizon reaches Bob only at the event horizon. 


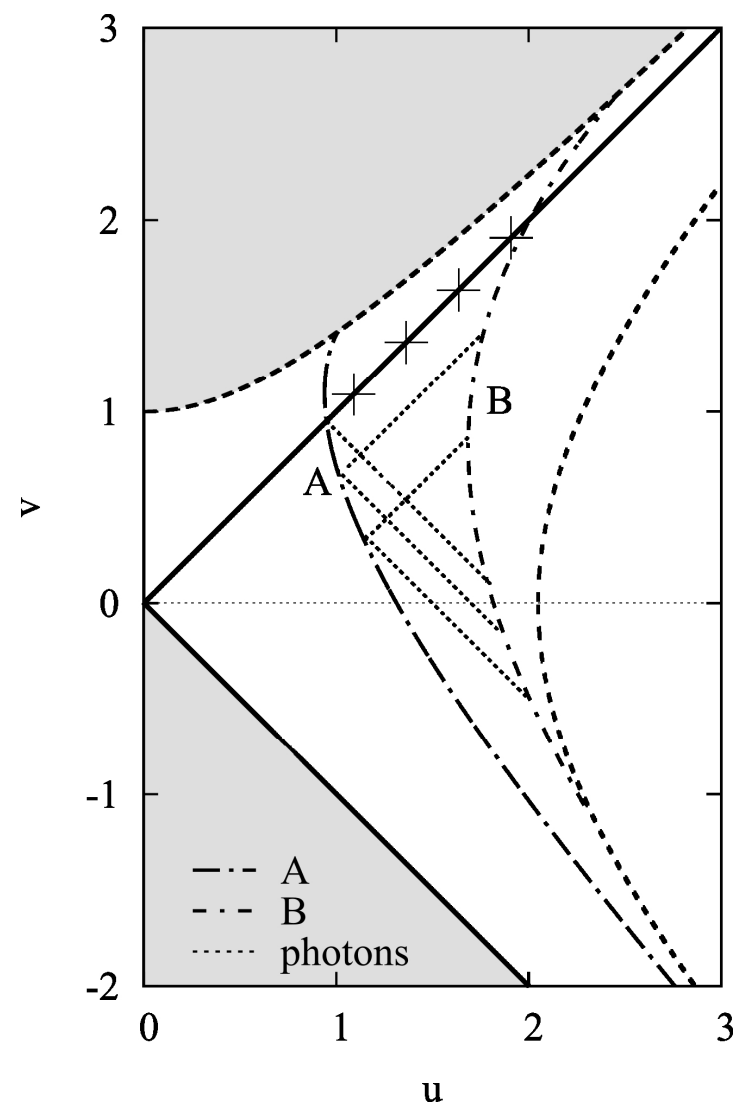

Fig.4 Bob receives signals from Alice until he, himself reaches the event horizon. This is an asymptotic process as no signal can be emitted at the horizon (there can be no "trapped" signal)

This is obviously a counter-intuitive outcome: it seems that Bob, approaching the event horizon can see Alice all the time, and moreover, that he is going to collide with her.

\section{Discussion and remarks}

Geodesic radial fall in Schwarzschild spacetime does not seem to yield any controversies, at least above the event horizon. Nevertheless, despite being an exactly solvable problem, it still reveals interesting behaviour - namely, the dramatic discrepancy between a rest observer's and an infalling observer's perception of the time taken to reach the event horizon. A manifestation of this discrepancy is the disruption of communication: an infalling observer continues to receive electromagnetic signals from a rest observer positioned above the event horizon, even when they drop below the event horizon (i.e. within the interior of a black hole). On the other hand, the rest observer can only receive signals from the infalling observer while the latter remains above the event horizon. As may be deduced from the above, this does not lead to an information paradox: the infalling observer, from the perspective of the rest observer, disappears into the latter's infinite future. It should be pointed out though, that Alice appears to take forever to reach $r_{S}$. So intuitively one may expect that light rays, sent by MS at any future coordinate time, will reach her before she crosses the horizon. In fact, this is not the case. Only signals sent up to $t=t_{1}$ will do so, and responses to these may be received in the limit of the limit of infinite coordinate time. And that is a direct consequence of the aforementioned divergence of time perception. 
However, as discussed in this paper, it is both interesting and instructive to explore another perspective - what will be perceived by a second infalling observer, Bob who follows a first infalling one, Alice? Bob reaches the event horizon in a finite time, so for him the argument of the infinite future does not apply. The analysis of observation made by Bob has been described here in a very qualitative manner, suitable for pedagogic purposes, by means of Kruskal coordinates. Paradoxically, those coordinates, devised mainly to match the interior of the black hole with its exterior, have been used here solely above the event horizon in order to illuminate the issue. The issue itself turns out to be the following: Bob continues to receive signals until the very last instant before crossing the event horizon. It would appear to him that he is going to collide with Alice. More precisely, it would appear that Bob's recording location approaches Alice's emission location; eventually overlapping on the event horizon itself. Hence the allusion in the title of this paper to touching ghosts.

It should be noted that this conclusion is, in a sense, inevitable. Bob, in chasing Alice, has to receive signals until he himself crosses the event horizon. This may be proven by considering a counterexample. Suppose that Bob noticed that Alice had already crossed the event horizon - this means that he is no longer receiving signals from her. Then he could emit a signal back to observers at rest letting them know that Alice had crossed the event horizon, and this signal would be received at a finite coordinate time. But this would then lead to a contradiction, since Alice's crossing of the event horizon cannot be recorded at a finite coordinate time as noted in the discussion above.

Although it would have been possible to draw this conclusion directly as noted above, it is more natural and possesses greater pedagogic value to do it in the deductive manner we have followed in this paper.

What happens at the event horizon and what happens below the event horizon? These questions are beyond the scope of the current discussion, but one can provide here two impressions resulting from studies undertaken elsewhere. First, it seems that one can not emit an outgoing signal, at the event horizon; such a signal would be trapped at the horizon. Second, it appears that in the case of Schwarzschild spacetime, the black hole interior represents a region where there are two possible communication scenarios. In one of these scenarios, signals emitted by Alice "towards" Bob - "outgoing" signals - are recorded by Bob below their emission location. This is in a sense an extension of the above discussion. The other scenario of communication in the black hole interior seems to be even more exotic.

The fascinating consequences of this for interaction between real objects provide grounds for further discussion.

\section{References}

[1] Hartle J B 2003 Gravity: An introduction to Einstein's General Relativity (Ch 9)Addison Wesley

[2] Misner Ch W, Thorne K S and Wheeler J A 1972 Gravitation, (Ch.25) W.H. Freeman

[3] Kruskal M D 1960 Phys. Rev. 119, 1743

Szekeres G 1960 Publ. Mat. Debrecen 7, 285

[4] Grib A A and Pavlov Yu V 2009 Is it possible to see the infinite future of the

Universe when falling into a black hole? arXiv:0906.1442[gr-qc]. 
[5] Schwarzschild K. 1916 Sitz. Preuss. Akad. Wiss. Berlin, 189

[6] Reissner H. 1916 Ann. der Phys. 355, 106

Nordström G. 1918 Kon. Nederland. Akad. Wet. Proc. 20, 1238

[7] Kerr R P 1963 Phys. Rev. Lett. 20, 237

[8] Newman E T et al. 1965 J. Math. Phys. 6, 918

[9] Frolov V P and Novikov I D 1998 Black Hole Physics: Basic Concepts and New Developments Dordrecht: Kluwer

[10] Hawking S. 2001 The Universe in a Nutshell New York: Bantam Books

[11] Chandrasekhar S 1983 The Mathematical Theory of Black Holes Oxford: Oxford Univ. Press

[12] Hawking S W and Ellis G F R 1973 The Large Scale Structure of Space-Time Cambridge: Cambridge Univ. Press

[13] Weinberg S 1972 Gravitation and Cosmology: Principles and Applications of the General Theory of Relativity Wiley

[14] Okun L B, Selivanov K G and Telegdi V L 2000 Am. J. Phys. 68, 115

[15] Lindquist R W and Wheeler J A 1957 Rev. Mod. Phys. 29, 432

[16] Narlikar J V Am. J. Phys. 62, 903 (1994)

[17] Radosz A, Augousti A T and Ostasiewicz K 2009 Phys. Lett. A 373, 801

[18] Augousti A T, Radosz A and Ostasiewicz K 2011 Eur. J. Phys. 32, 535 